\title{
Using Acellular Dermal Matrix to Treat Repetitive Tendon Rupture after Excision of Ruptured Intratendinous Ganglion
}

\author{
Sung-Min Sohn (D), Na-Hyun Hwang (D), Jae-Ho Chung (D), Seung-Ha Park (D), Eul-Sik Yoon (D) \\ Department of Plastic and Reconstructive Surgery, Korea University Anam Hospital, Seoul, Korea
}

\begin{abstract}
Existing literature has not explored repeated tendon ruptures caused by torn intratendinous ganglions. The treatment of intratendinous ganglion of the hand remains a challenge, as it is difficult to completely excise the ganglion while also preserving the tendon. Herein is a report on a case of ruptured intratendinous ganglion in the extensor digitorum communis which resulted in repeated tendon reconstruction surgeries. The authors incorporated an acellular dermal matrix with tendon transfer, demonstrating great clinical improvement and successful repair thus far.
\end{abstract}

Keywords: Ganglion cysts; Extensor digitorum communis; Acellular dermal matrix

\section{Introduction}

A ganglion cyst is a well-known benign lesion composed of mucinous fluid. The degeneration of periarticular components such as joint capsule, tendon, or tendon sheath is associated with its formation [1]. While ganglion cysts occur most commonly near the joints of the wrist bones on the dorsal side, intratendinous ganglions are uncommon and have rarely been reported [2]. Furthermore, there were no reported cases of tendon ruptures caused by ruptured intratendinous ganglion cysts. The study herein reports a case of repetitive tendon rupture after excision of intratendinous ganglion that developed in the extensor digitorum communis (EDC) of the right hand.

The patient consented to the disclosure of his course of treatment, including personal medical information and clinical photos, both in verbal and written form. We conducted our investigation according to the principles expressed in the World Medical Association Declaration of Helsinki (June 1964) and subsequent amendments.

\section{Case}

A 66-year-old male patient visited the clinic with spontaneous limitation of extension on his right little finger (Fig. 1). Suffering from chronic renal failure due to a kidney tumor, multiple IV cannulations were carried out on the dorsum of his right hand during hemodialysis. Prior to visiting our hospital, the patient had received a kidney transplant and had been on immunosuppressive drugs. Upon physical examination, a painless movable $1 \times 0.5 \mathrm{~cm}$-sized mass was present on the dorsum of his right wrist.

\section{Case Report}

Received: October 17, 2020

Revised: December 22, 2020

Accepted: December 27, 2020

Corresponding author:

Eul-Sik Yoon, M.D., Ph.D.

Department of Plastic and Reconstructive Surgery, Korea University Anam Hospital, 73 Inchon-ro, Seongbuk-gu, Seoul 02841 Korea

Tel: +82-2-920-5368

Fax: +82-2-922-7437

E-mail: yesanam2@korea.ac.kr

This is an Open Access article distributed under the terms of the Creative Commons Attribution Non-Commercial License (https://creativecommons.org/licenses/by-nc/4.0/) which permits unrestricted non-commercial use, distribution, and reproduction in any medium, provided the original work is properly cited.

(c) 2021 Korean Wound Management Society 


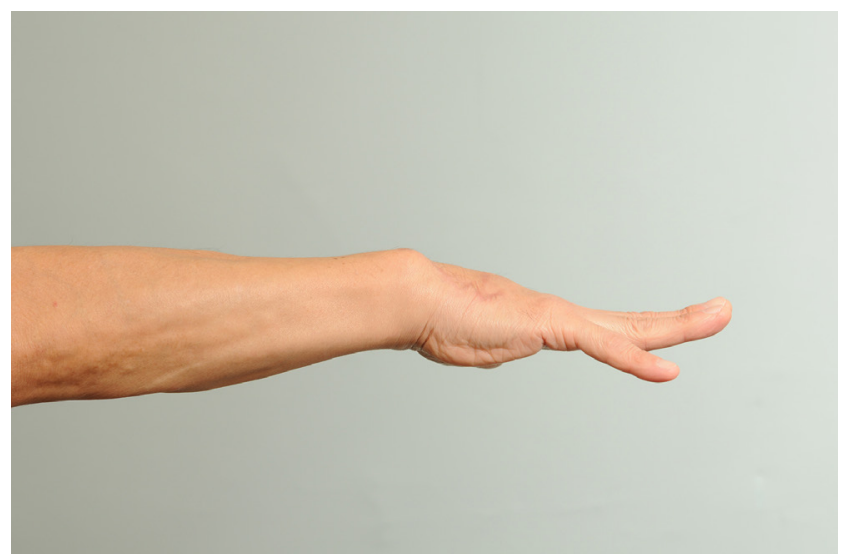

Fig. 1. Initial preoperative photo. The patient was unable to fully extend his little finger.
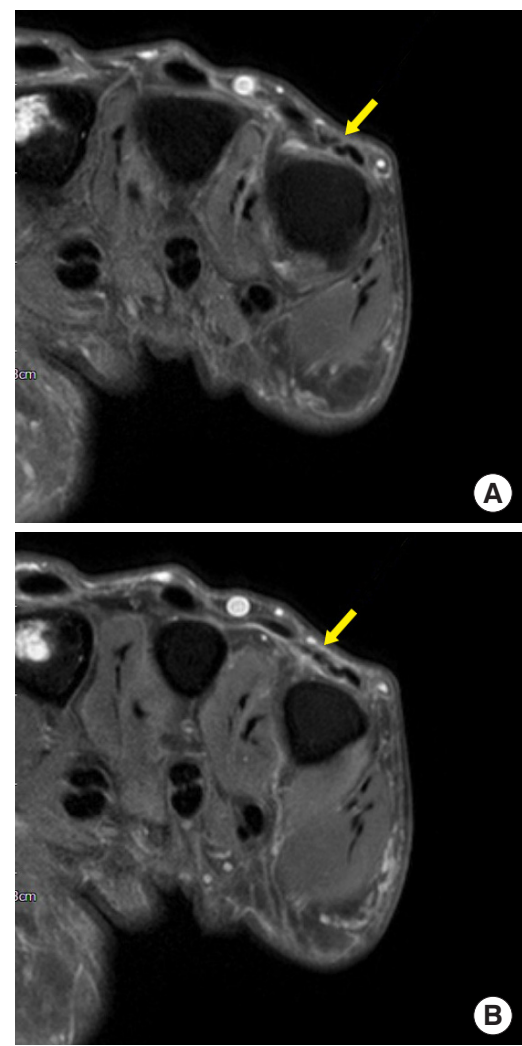

Fig. 2. Magnetic resonance imaging of right hand. Axial proton density spectral presaturation inversion recovery images show partial division of the fifth extensor digitorum communis (arrow) (A) and edema around the tendon (arrow) (B).

Magnetic resonance imaging showed partial division of the fifth EDC and edema around the tendon (Fig. 2). Based on these findings, a ganglion was suspected and surgical exploration was performed. A ruptured cystic nodule was found in the fifth EDC tendon, and slight tenosynovitis was observed

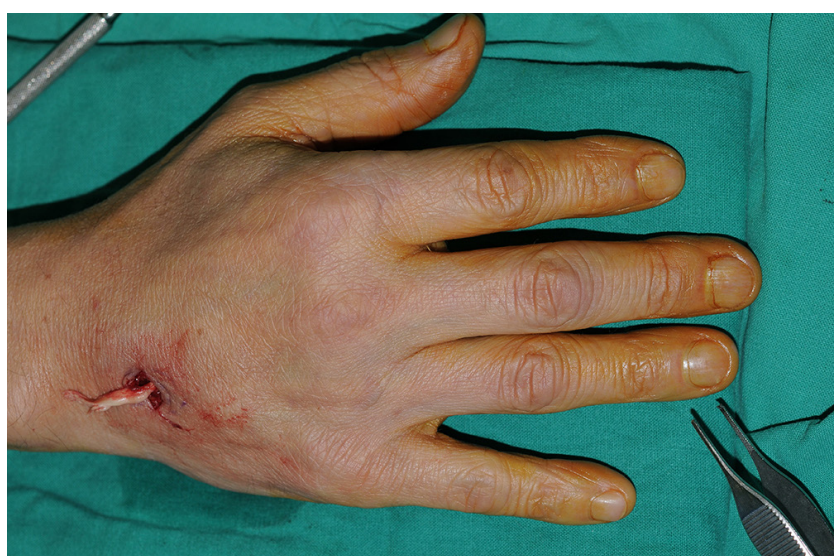

Fig. 3. Initial intraoperative photo. A tendon defect was left at the proximal end of the fifth extensor digitorum communis after ganglion cyst excision.

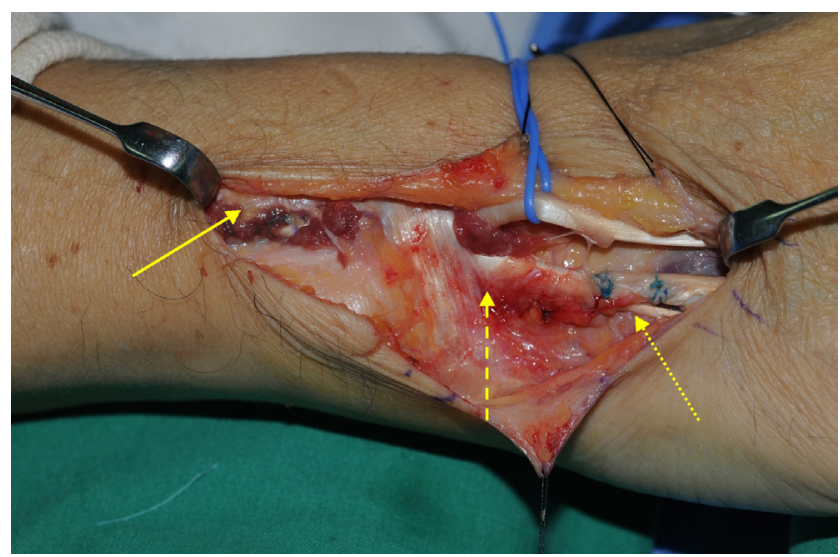

Fig. 4. Initial repair of ruptured tendon. Approximation of the fifth extensor digitorum communis (EDC) to the side of the fourth EDC using a palmaris longus tendon graft. The fourth EDC (arrow), the fifth EDC (dotted arrow), the palmaris longus graft (dashed arrow) are indicated.

around the impinged tendon. Tendon injury was found around the cystic nodule. The nodule was cautiously excised to preserve the underlying tendon as much as possible (Fig. 3). Despite efforts for treatment, tendon division was observed after the excision and reconstruction of the tendon with tenorrhaphy was carried out. Histopathology of the nodule revealed a ganglion cyst.

At 1 month postoperative, reconstructive surgery was planned since there was no clinical improvement on the finger extension on his little finger. A total division of the EDC was discovered on the right fifth finger at the metacarpal shaft level. Adhesion was severe on both the proximal and distal parts, making it difficult to connect the two ends. As a solution, a palmaris longus tendon graft was performed on the severed 


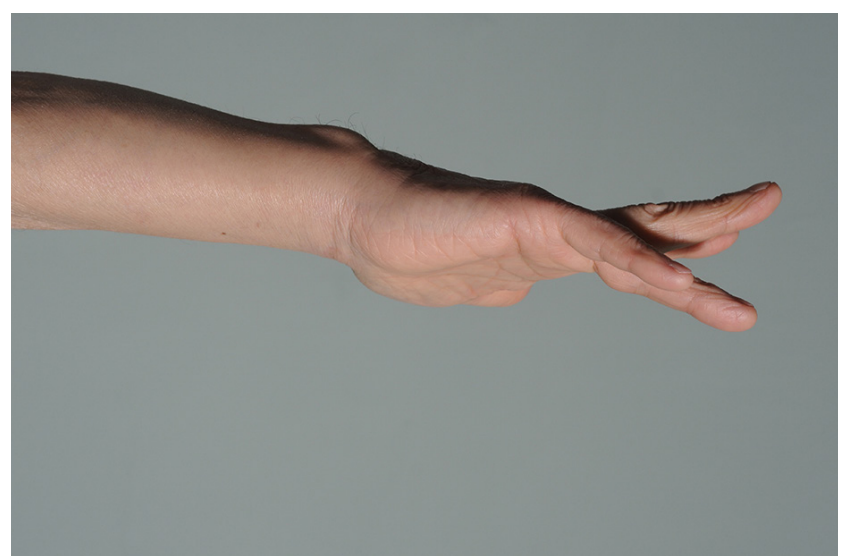

Fig. 5. Clinical photo taken at 5 months postoperative. Extension limitation was shown on the fourth and fifth fingers.

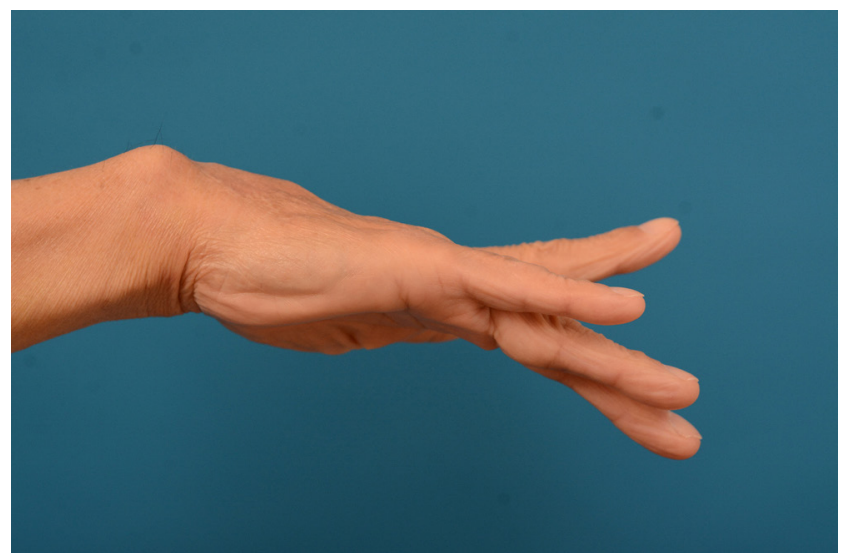

Fig. 6. Clinical photo taken at 6 years postoperative. Decreased motor and extension on the third finger were observed.

ends of the fifth EDC to the fourth EDC in an end-to-side manner (Fig. 4). Each end was sutured with the Pulvertaft technique using 4-0 Ethibond. After the surgery, an extensor splint was applied for $4-5$ weeks and, for the following 2-3 weeks, nighttime splints were recommended, while during the daytime, the patient was instructed to actively exercise his hand, albeit with caution. Until 3 months postoperative, slight improvement on the movement of the fifth finger was observed.

At 5 months postoperative, the patient showed lack of extension on his right fourth and fifth fingers (Fig. 5). Surgical intervention revealed total division of the fourth EDC. After completion of adhesiolysis, tenorrhaphy was carried out by approximation of the distal end of the fourth EDC to the side of the third EDC. The third EDC was split into two strands and was sutured with fourth EDC using the Pulvertaft technique.
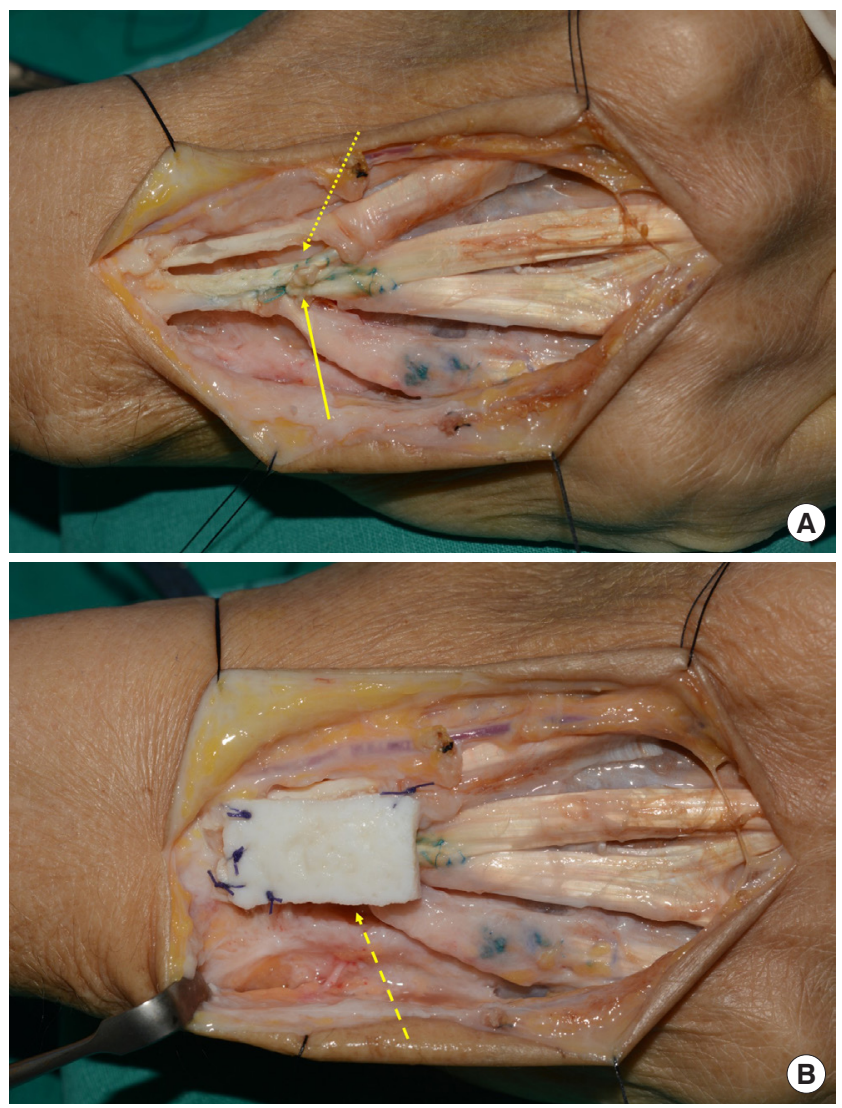

Fig. 7. Tendon reconstruction with tendon transfer and ADM application. (A) The distal end of the third EDC (arrow) was transferred to the side of the second EDC (dotted arrow). (B) ADM tailored (dashed arrow) and placed over the repaired tendons. ADM, acellular dermal matrix; EDC, extensor digitorum communis.

Approximately 6 years after the last surgery, re-rupture occurred at the previously reconstructed third EDC (Fig. 6). The patient showed motor decrease on his right third and fourth fingers and surgical intervention was scheduled again. The previously transferred tendons were intact, but the third EDC was worn out at a more proximal site. An end-to-end tenorrhaphy was infeasible. The distal part of the third EDC was transferred to the side of the second EDC and the same surgical technique was used to suture the tendons (Fig. 7A). In addition, acellular dermal matrix (ADM; MegaDerm, L\&C BIO Inc., Seongnam, Korea) was tailored $(1 \times 2 \mathrm{~cm} ; 3.0 \mathrm{~mm}$ in thickness) to augment tendon repair and to minimize tendon adhesion (Fig. 7B). The ADM was incorporated as an "on-lay" on top of the repaired tendons. Lastly, we carefully covered the tendon sheath over the ADM.

At 7 years and 6 months since the first operation, the patient's progress was satisfactory with a nearly full range of motion in all five digits (Fig. 8). 


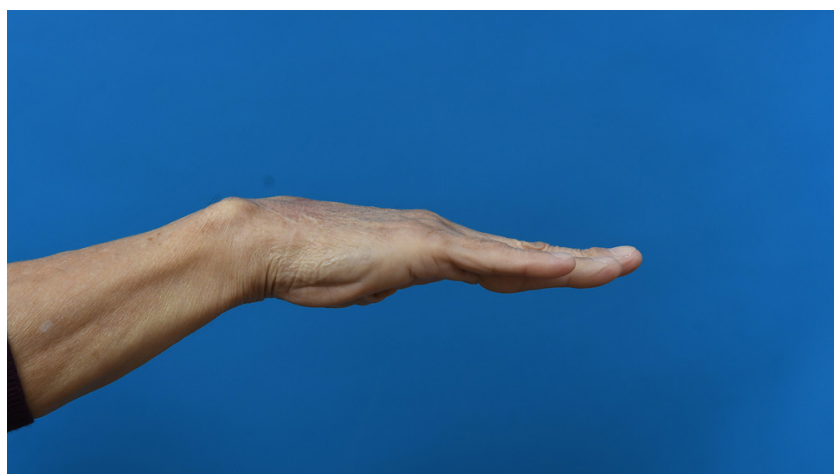

Fig. 8. Postoperative photo after 7 years and 6 months. Patient showing extension on all five fingers.

\section{Discussion}

Intratendinous ganglion cyst of the hand is rarely found. Botchu et al. [3] previously reported an intratendinous ganglion cyst of the extensor digitorum tendon. The reported patient went through chemotherapy and received multiple IV cannulations. The authors of that study postulated that the ganglion cyst had occurred as a result of tendon injuries during repetitive venipunctures. Similarly, the patient in the current study received hemodialysis and venipunctures, which were performed frequently on both dorsal hands. This suggests that the tendons might have been injured with a similar pathogenesis which led to the development of the intratendinous ganglion.

To this date, there is no consensus about the treatment method for intratendinous ganglion of the hand. Seidman and Margles [4] stated that excision of the ganglion is necessary because of its weakening of the tendon. Meanwhile, some studies suggested an ultrasound-guided aspiration as an effective therapeutic modality [5]. While all methods above have shown some promising results, recurrence after surgery due to incomplete removal still remains a major complication [4].

Reconstructing an injured extensor tendon is also a difficult process since the structure of the extensor tendons of the hand is rather complex [6]. Because extensor tendons are connected through juncturae tendinums, a slight change to the extensor tendon may affect the motion of the entire hand [7]. The task becomes more challenging when there are defects or loss in the tendons. In our case, the fourth and third EDC tendons were sequentially damaged after the reconstruction of the fifth EDC tendon. Following such rupture, reconstruction of the tendon is complicated, often with suboptimal functional outcomes. Treatment methods for tendon injuries caused by atraumatic causes such as rheumatoid arthritis have been previously reported [8]. Since inflammation is the main culprit in most cases, the recommended approaches in other studies were also applicable to this case study.

More diverse indications for tendon transfer mean the procedure is now widely performed when primary repair seems impossible [6,9]. Schindele et al. [8] summarized the preferred treatment for multiple tendon rupture scenarios. For instance, if the extensor digiti minimi (EDM) tendon is the only tendon injured, it can be easily reconstructed by end-to-side suture to the fifth or fourth EDC. With combined rupture of EDM and the fifth EDC, tendon transfer to the ring finger EDC is mainly used. To prevent forced abduction of the fifth finger after reconstruction, an extensor indicis proprius to the fifth EDC/ EDM tendon transfer is considered when rupture is located distal to the mid-metacarpal level.

Unfortunately, in our case tendon transfer alone was not sufficient to salvage the tendons. The tensile strength was weakened, and the direction of pull for those tendons was also altered after multiple transfers. Moreover, owing to a history of kidney transplantation and the use of immunosuppressive drugs, it is possible that the reconstructed tendons were not able to sustain a normal healing process. In a study conducted by Jones et al. [10], the authors mentioned the negative effects of chronic intake of immunosuppressants on the tendons in the hand. They also pointed out that the hands of kidney transplantation patients are vulnerable to arterial ischemia because of arteriosclerosis or steal phenomenon. Our study therefore gradually focused not only on the tendons but also on the surrounding tissues. This was also important because excursion of the tendon closely involves the interconnection between the tendon and soft tissues. Satonaka et al. [11] proved the close relationship by revealing histopathological findings of a tendon adjacent to a ganglion cyst showing degenerative change and an accumulation of hyperplastic synovial cells.

To address the many complexities, ADM was applied over the reconstructed tendons. $\mathrm{ADM}$ is known to improve healing by incorporating reepithelization, neovascularization, and cell infiltration around the tendon-graft area [12]. ADM can also reinforce tendon strength by offering mechanical support [13]. Application of ADM in previous studies focused on tendons in the lower extremities or larger tendons of the body. Bertasi et al. [14] introduced a successful case of ADM usage in Achilles tendon reconstruction and studied the cellular change in the graft-paratenon interface. They concluded with recellular- 
ization and revascularization as the mechanism for integration. In another study, Acevedo et al. [15] stated that ADM was intended for use in an on-lay method, which maximizes incorporation. The surgical techniques of applying the ADM to the tendons in this case study were based on these studies. The aim was to ultimately obtain a well-constructed, integrated structure.

Existing literature did not explore ADM application on the tendons of the finger. In this study, ADM was used to promote tendon repair and to overcome repetitive extensor tendon rupture. Considerable progress was observed with no further tendon ruptures. Though results from this study's single patient cannot be generalized, the findings may aid other surgeons using the ADM for tendon reconstruction in the hand. While it is necessary to observe a larger population for a more comprehensive investigation, the fact that revisional surgery is not standard practice in tendon repair may limit a larger study. Repeated tendon ruptures may be prevented in the future if the "straight line-pull" principle is observed during tendon transfer. Nevertheless, the preliminary results of using ADM during extensor tendon reconstruction in the hand suggest that the method is safe and without re-ruptures or complications.

\section{Conflict of interest}

No potential conflict of interest relevant to this article was reported.

\section{ORCID iDs}

$\begin{array}{ll}\text { Sung-Min Sohn } & \text { https://orcid.org/0000-0001-8731-0121 } \\ \text { Na-Hyun Hwang } & \text { https://orcid.org/0000-0001-8784-7383 } \\ \text { Jae-Ho Chung } & \text { https://orcid.org/0000-0002-8351-2444 } \\ \text { Seung-Ha Park } & \text { https://orcid.org/0000-0002-2667-2791 } \\ \text { Eul-Sik Yoon } & \text { https://orcid.org/0000-0001-5734-6625 }\end{array}$

\section{References}

1. McCarthy CL, McNally EG. The MRI appearance of cystic lesions around the knee. Skeletal Radiol 2004;33:187-209.

2. Kulinski S, Gutkowska O, Mizia S, et al. Ganglions of the hand and wrist: retrospective statistical analysis of 520 cas- es. Adv Clin Exp Med 2017;26:95-100.

3. Botchu R, Bharath A, Uhiara O, et al. Iatrogenic intratendinous ganglion cyst of the extensor digitorum tendon following intravenous cannulation. J Ultrasound 2018;21:32931.

4. Seidman GD, Margles SW. Intratendinous ganglia of the hand. J Hand Surg Am 1993;18:707-10.

5. Jose J, Silverman E, Kaplan L. Symptomatic ganglion cyst of the popliteus tendon treated with ultrasound-guided aspiration and steroid injection: a case report. Sports Health 2011;3:393-5.

6. Sammer DM, Chung KC. Tendon transfers: part I. principles of transfer and transfers for radial nerve palsy. Plast Reconstr Surg 2009;123:169e-177e.

7. von Schroeder HP, Botte MJ. The functional significance of the long extensors and juncturae tendinum in finger extension. J Hand Surg Am 1993;18:641-7.

8. Schindele SF, Herren DB, Simmen BR. Tendon reconstruction for the rheumatoid hand. Hand Clin 2011;27:105-13.

9. de Roode CP, James MA, Van Heest AE. Tendon transfers and releases for the forearm, wrist, and hand in spastic hemiplegic cerebral palsy. Tech Hand Up Extrem Surg 2010;14:129-34.

10. Jones NF, Friedman AC, Khiabani KT. Hand surgery in organ transplant patients. J Hand Surg Am 2004;29:154-8.

11. Satonaka H, Tsujii M, Sudo A. Tenosynovitis of the extensor pollicis longus tendon caused by an intratendinous ganglion: a case report. J Hand Surg Eur Vol 2014;39:66971.

12. Capito AE, Tholpady SS, Agrawal H, et al. Evaluation of host tissue integration, revascularization, and cellular infiltration within various dermal substrates. Ann Plast Surg 2012;68:495-500.

13. Barber FA, Burns JP, Deutsch A, et al. A prospective, randomized evaluation of acellular human dermal matrix augmentation for arthroscopic rotator cuff repair. Arthroscopy 2012;28:8-15.

14. Bertasi G, Cole W, Samsell B, et al. Biological incorporation of human acellular dermal matrix used in Achilles tendon repair. Cell Tissue Bank 2017;18:403-11.

15. Acevedo DC, Shore B, Mirzayan R. Orthopedic applications of acellular human dermal allograft for shoulder and elbow surgery. Orthop Clin North Am 2015;46:377-88. 